\title{
Contactless Ultrasound Generation in a Crucible
}

\section{Valdis Bojarevics, Georgi S. Djambazov \& Koulis A. Pericleous}

Metallurgical and Materials Transactions A

ISSN 1073-5623

Volume 46

Number 7

Metall and Mat Trans A (2015) 46:2884-2892

DOI 10.1007/s11661-015-2824-5

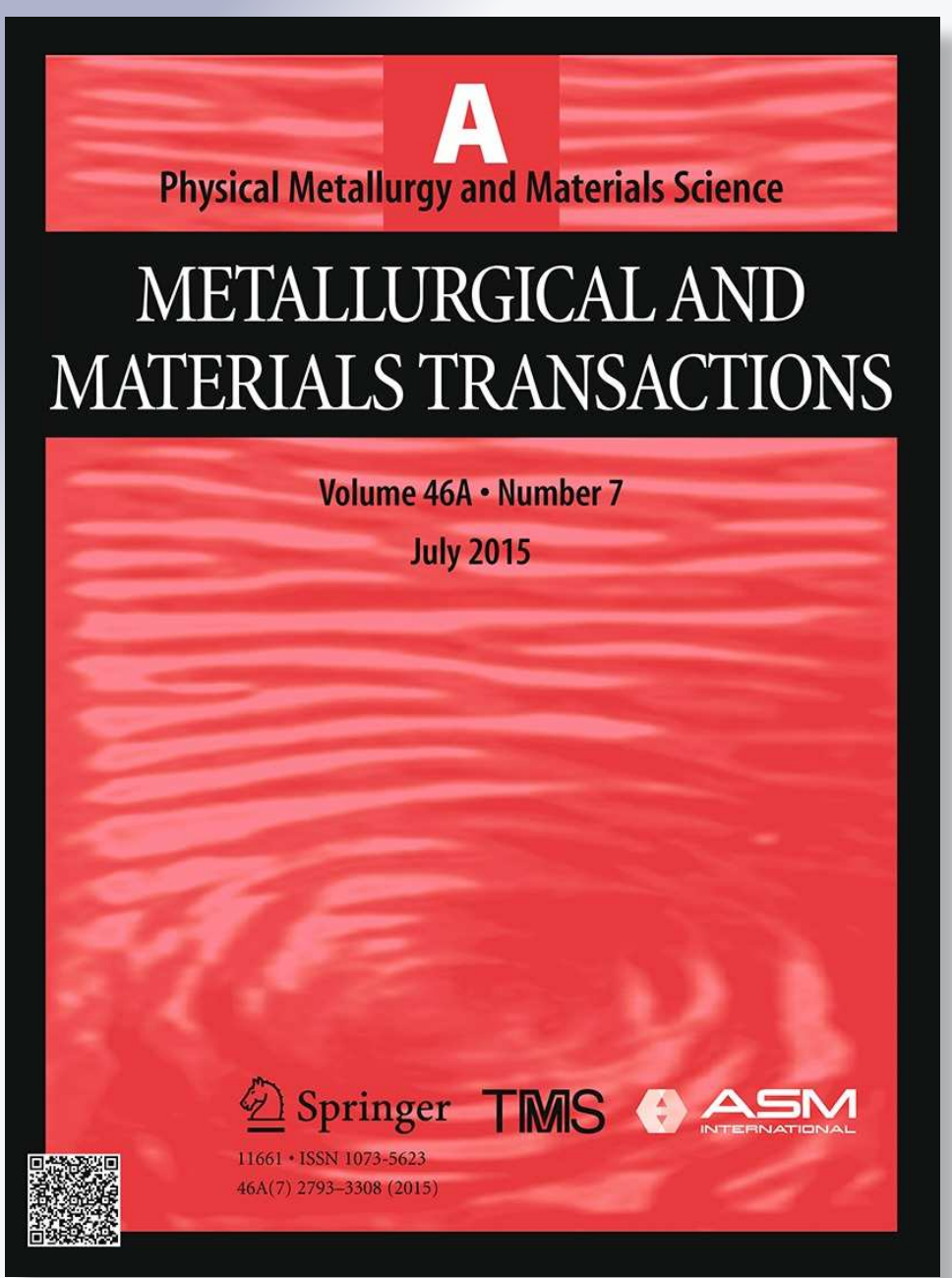

㩏 Springer 
Your article is protected by copyright and all rights are held exclusively by The Minerals, Metals \& Materials Society and ASM International. This e-offprint is for personal use only and shall not be self-archived in electronic repositories. If you wish to selfarchive your article, please use the accepted manuscript version for posting on your own website. You may further deposit the accepted manuscript version in any repository, provided it is only made publicly available 12 months after official publication or later and provided acknowledgement is given to the original source of publication and a link is inserted to the published article on Springer's website. The link must be accompanied by the following text: "The final publication is available at link.springer.com". 


\title{
Contactless Ultrasound Generation in a Crucible
}

\author{
VALDIS BOJAREVICS, GEORGI S. DJAMBAZOV, and KOULIS A. PERICLEOUS
}

\begin{abstract}
Ultrasound treatment is used in light alloys during solidification to refine microstructure, remove gas, or disperse immersed particles. A mechanical sonotrode immersed in the melt is most effective when probe tip vibrations lead to cavitation. Liquid contact with the probe can be problematic for high temperature or reactive melts leading to contamination. An alternative contactless method of generating ultrasonic waves is proposed, using electromagnetic (EM) induction. As a bonus, the EM force induces vigorous stirring distributing the effect to treat larger volumes of material. In a typical application, the induction coil surrounding the crucible - also used to melt the alloy - may be adopted for this purpose with suitable tuning. Alternatively, a top coil, immersed in the melt (but still contactless due to EM force repulsion) may be used. Numerical simulations of sound, flow, and EM fields suggest that large pressure amplitudes leading to cavitation may be achievable with this method.
\end{abstract}

DOI: $10.1007 / \mathrm{s} 11661-015-2824-5$

(C) The Minerals, Metals \& Materials Society and ASM International 2015

\section{INTRODUCTION}

INTENSE vibration of the liquid metal during solidification is known to refine microstructure, remove trapped gas, and reduce porosity. ${ }^{[1-3]}$ Vibration can be achieved through various means: shaking the crucible, inserting a mechanical stirrer in the melt or, more commonly, using an ultrasonic horn e.g., in the DC casting of aluminum ingots. ${ }^{[3]}$ In ultrasonic processing, frequencies around $20 \mathrm{kHz}$ were found to be the most effective,$^{[3]}$ a fact attributed to the onset of cavitation. Gas micro-bubbles exist in the liquid either due to dissolved gases coming out of solution as the melt cools (e.g., $\mathrm{H}_{2}$ in $\mathrm{Al}$ ), air trapped with oxides during mold filling, or gas attached to the surface of immersed particles. Bubbles, subjected to an imposed pressure sine-wave, first expand to many times their original size and then collapse violently generating high speed microjets and shock waves. These events fragment emerging dendrites, or facilitate the breakup and dispersion of particle clusters to generate nucleation sites. Evidence of cavitation has been seen in situ recently, ${ }^{[4]}$ found in postsolidification analyses and further shown to depend on a critical pressure threshold. ${ }^{[3]}$ Of relevance to the present study, ultrasonic processing is one of the candidate techniques for the production of MMNCs.

There are, however, several disadvantages in immersive stirring/vibration techniques: contact with the liquid metal leads to contamination of the melt and conversely erosion of the immersed probe surface, requiring frequent replacement. These problems are limiting when the technology is to be applied to high temperature alloys

VALDIS BOJAREVICS, Reader in Computational MHD, GEORGI S. DJAMBAZOV, Senior Research Fellow, and KOULIS A. PERICLEOUS, Professor of Computational Fluid Dynamics, CoDirector, are with the Centre for Numerical Modelling and Process Analysis, University of Greenwich, London SE10 9LS, UK. Contact email: k.pericleous@gre.ac.uk

Manuscript submitted December 19, 2014.

Article published online March 18, 2015
(ODS steel, nickel superalloys) or to highly reactive metals (Ti, Zr). Another limiting factor of the immersed sonotrode approach is the localized effect of the cavitation region, which leads to long processing times, high cost/energy usage and consequently small treatment volumes. EM induction is an attractive alternative. It provides melt stirring and heat. What is overlooked is the ability of the AC component of the induced Lorentz force to generate a strong sound field within the melt, equivalent to that produced by an immersed sonotrode, but without contact. Vives ${ }^{[5]}$ was the first to investigate experimentally such a non-contact technique, using a combination of static and AC magnetic fields. Other investigators followed this lead, used different configurations; for AlSi hypereutectic alloys, ${ }^{[3,6]}$ for gray iron melts and aluminum alloys. ${ }^{[7]}$ In each case, different thresholds of pressure amplitude were found to be necessary and a range of frequencies was used from 50 to $50 \mathrm{kHz}$. There has been no systematic study of the sound field generated in these situations, which led us in a previous publication, ${ }^{[8]}$ to examine the sound field generated by the cylindrical induction coil in an induction crucible, coupling the compressible sound wave equations with the pressure source generated by the electromagnetic field and the Rayleigh-Plesset equations for cavitating bubbles. This simulation demonstrated that pressure amplitudes likely to cause cavitation of dissolved gas can be generated, provided the AC frequency is tuned to approach wave resonant conditions-a similar conclusion was reached by Vives, who considered the design of a resonant EM cavity reproduced in Figure 1 for this purpose.$^{[5]}$ In the present contribution, we extend this idea via the introduction of a tuned top coil* gradually inserted

*VibroEM patent application \#PCT/EP2013/067896.

into the liquid metal volume, but nevertheless still using EM repulsion to prevent contact with the metal. In this very close proximity to the surface, intense vibrations and 
melt stirring can be produced which can be controlled via the depth of immersion, current magnitude, and frequency. The concept is tested theoretically in this contribution through a coupled MHD-acoustic numerical simulation. Experimental implementation is currently in progress and results will follow in a future publication.

The remainder of the paper is arranged as follows: The problem to be addressed is described, followed by the sets of coupled equations describing the flow, heat transfer, magnetic and sound fields. Illustrative results of simulations using aluminum melts are then given, followed by discussion and conclusions.

\section{PROBLEM SETUP}

A typical ceramic induction crucible is shown in Figure 2, together with two alternative EM excitation coil arrangements. The cylindrical induction coil and the

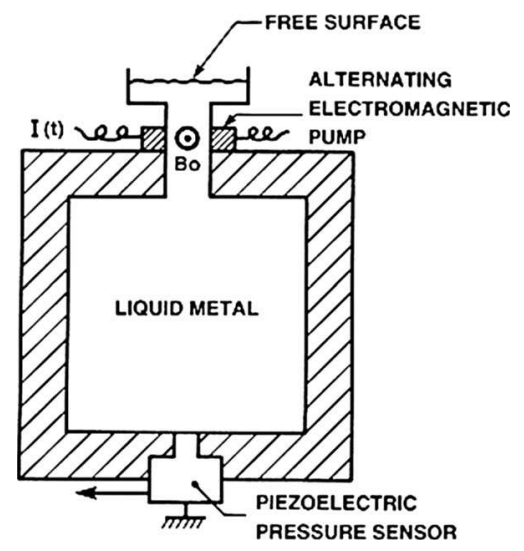

Fig. 1-A resonant EM cavity idea, reproduced from Vives. ${ }^{[5]}$

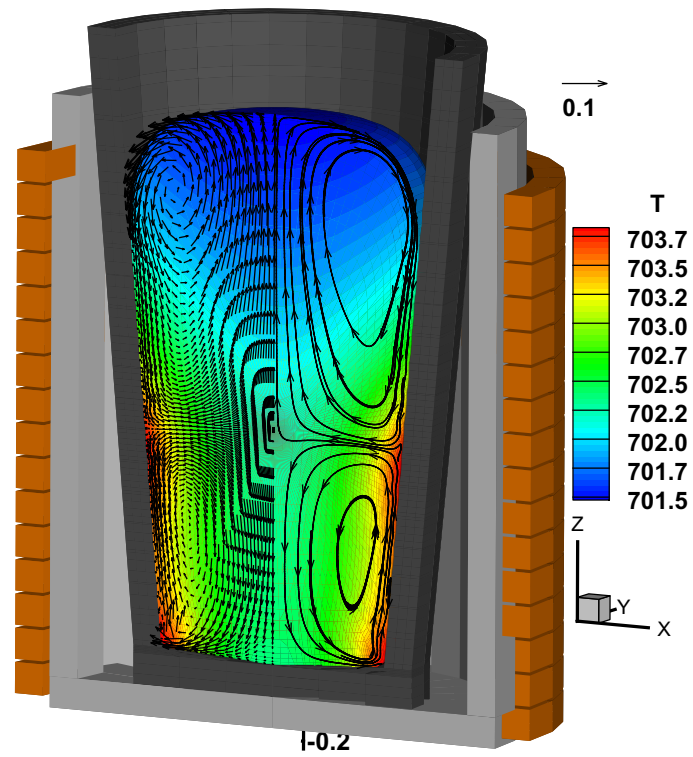

top coil can be operated independently of each other and at different frequencies. The function of the cylindrical coil remains that of heating and stirring, while the top coil is there to provide controlled vibration. The top coil is allowed to move vertically so that it can gradually enter the melt, forming a depression in the free surface. In reality, both coils contribute to the sound field within the melt. The ceramic crucible is enclosed in a graphite container that is itself surrounded by the coil. The crucible is filled with aluminum.

The flow and thermal fields resulting from the EM force and Joule heating are solved using an incompressible spectral collocation technique, assuming harmonic averaging for mean values as described in previous publications. ${ }^{[9,10]}$ Time accurate information is extracted within each $\mathrm{AC}$ cycle once a pseudo-steady condition is reached, to enable the driving sound source evaluation for the compressible acoustic simulation.

\section{MODELING APPROACH}

Due to the different timescales involved, the mean flow and sound propagation problems are treated in sequence, in the following fashion:

(i) The mean flow is computed until pseudo-steady conditions are reached, with a timestep dictated by the need to resolve inertial flow oscillations and solidification dynamics. (ii) This is followed by much shorter timesteps that resolve EM Lorentz force oscillations within the driving frequency period. (iii) In steps (i)-(ii), the problem is assumed incompressible; for sound field calculations, compressibility is introduced and the wave equations are solved with the body forces computed in (ii) acting as boundary conditions providing the source

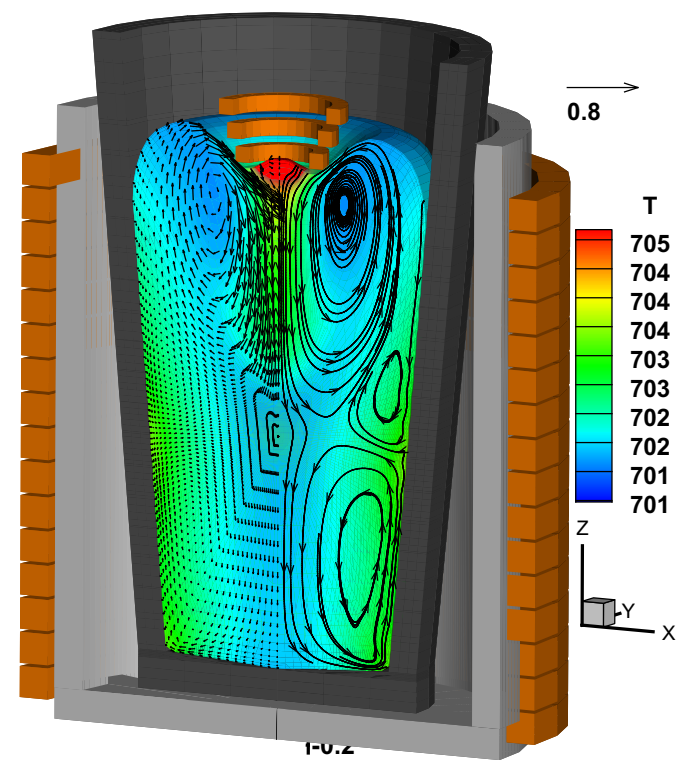

Fig. 2-Two alternative arrangements for melt stirring and vibration of the melt. The computed velocity and temperature fields are shown, with the LHS figure representing a traditional arrangement with a cylindrical coil $(2 \mathrm{kHz}, 1.6 \mathrm{kA})$; the RHS figure depicts the new concept of the immersed top coil operating at $10 \mathrm{kHz}$. 
of sound. (iv) The frequency and amplitude of the external excitation can be tuned to reach the pressure fluctuation levels where cavitation of any gas dissolved in the melt becomes a possibility. Where such a threshold is reached, a separate Rayleigh-Plesset type equation $^{[11]}$ may be used to determine the effects of cavitation on nucleation sites or on particle cluster break up. Step (iv) does not form part of this paper, details can be found instead in Lebon et al. ${ }^{[12]}$

\section{MHD FLOW AND HEAT TRANSFER}

The time-dependent conservation equations for mass, momentum, and energy are solved, accounting for the effects of electromagnetic interaction and change of phase. An in-house software code 'SPHINX' solves the equations numerically using a spectral collocation method on a continuously deformable mesh coincident with the metal volume as described in Pericleous et al. ${ }^{[9]}$ The method allows real-time deformation of the liquid free surface based on the balance of forces acting. A description of the main equations follows:

Momentum

$$
\begin{aligned}
\partial_{t} \mathbf{v}+(\mathbf{v} \cdot \nabla) \mathbf{v}= & -\rho^{-1} \nabla p+\nabla \cdot\left(v_{\mathrm{e}}\left(\nabla \mathbf{v}+\nabla \mathbf{v}^{\mathrm{T}}\right)\right) \\
& +\rho^{-1} \mathbf{f}_{\mathrm{m}}+S_{\mathrm{D}},
\end{aligned}
$$

where $\mathbf{f}_{\mathrm{m}}$ is the volumetric electromagnetic force and $S_{\mathrm{D}}$ a 'Darcy' resistance term introduced to account for the phase change 'mushy' zones and to stop flow in solidifying regions.

Continuity for an incompressible fluid

$$
\nabla \cdot \mathbf{v}=0
$$

The boundary conditions are the no-slip condition at the solid walls $(\mathbf{v}=0)$ or the free surface dynamic and kinematic conditions when the liquid metal is detached from the solid wall (see $e^{[9,10]}$ for details).

Energy conservation

$$
\frac{\partial\left(\rho C_{\mathrm{p}} T\right)}{\partial t}+\nabla\left(\rho C_{\mathrm{p}} v T\right)=\nabla\left(k_{\mathrm{eff}} \nabla(T)\right)+q_{\mathrm{e}}+q_{\mathrm{L}}
$$

The last two terms in Eq. [3] represent Joule heating, $q_{\mathrm{e}}$ and latent heat release, $q_{\mathrm{L}}$.

$$
q_{\mathrm{e}}=\frac{J^{2}}{\sigma} ; q_{\mathrm{L}}=-\frac{\partial}{\partial t}\left(\rho f_{\mathrm{L}} L\right)-\nabla \cdot\left(\rho \mathbf{v} f_{\mathrm{L}} L\right)
$$

The liquid fraction is defined as a function of $T$ :

$$
f_{\mathrm{L}}=\left\{\begin{array}{lll}
1 & T>T_{\mathrm{L}} & \text { liquid } \\
\left(\frac{T-T_{\mathrm{S}}}{T_{\mathrm{L}}-T_{\mathrm{S}}}\right) & T_{\mathrm{S}} \leq T \leq T_{\mathrm{L}} & \text { mushy zone } \\
0 & T<T_{\mathrm{S}} & \text { solid }
\end{array}\right.
$$

Then,

$$
S_{\mathrm{D}}=\frac{\mu}{K} \mathbf{v} ; \quad K=\frac{f_{\mathrm{L}}^{3}}{\zeta\left(1-f_{\mathrm{L}}\right)^{2}} .
$$

Heat transfer boundary conditions are stated for free surface radiation and wall loss, also described in Reference 10. The effective heat transfer at solid walls is then given by $-\rho C_{\mathrm{p}} \alpha_{\mathrm{e}} \partial_{n} T=h\left(T-T_{\mathrm{w}}\right)$, where $h(T)$ is the heat transfer coefficient, and at the free surface the radiation condition is used: $-\rho C_{\mathrm{p}} \alpha_{\mathrm{e}} \partial_{n} T=$ $\varepsilon \sigma_{\mathrm{b}}\left(T^{4}-T_{\mathrm{w}}^{4}\right)$, with $\alpha_{\mathrm{e}}$ being the turbulent thermal diffusion coefficient.

Turbulence is modeled using the $k-\omega$ model of Wilcox: ${ }^{[13]}$

$$
\left.\begin{array}{l}
\partial_{t} k+\mathbf{v} \cdot \nabla k=\nabla \cdot\left[\left(v+\sigma_{\mathrm{k}} v_{\mathrm{T}}\right) \nabla k\right]+G-\beta^{*} \omega k \\
\partial_{t} \omega+\mathbf{v} \cdot \nabla \omega=\nabla \cdot\left[\left(v+\sigma_{\omega} v_{\mathrm{T}}\right) \nabla \omega\right]+\alpha \frac{\omega}{k} G-\beta \omega^{2} \\
v_{\mathrm{T}}=\alpha^{*} \frac{k}{\omega}
\end{array}\right\},
$$

where $\omega$ is the frequency of vorticity fluctuations and $k$ the turbulence kinetic energy per unit mass, and the various constants are functions of $R_{\mathrm{T}}$, the local turbulent Reynolds number (see Wilcox ${ }^{[13]}$ ).

The electromagnetic field distribution is computed according to the mutual inductance algorithm described in detail in References 10, 14. The method was tested against analytic solutions and against experimental measurements e.g., in an In-Ga-Sn melt ${ }^{[14]}$ and elsewhere. The magnetic field $\mathbf{B}$ and the electric current at any time instant can be expressed analytically in terms of elliptic functions without the need for numerical differentiation, once the solution for the magnetic vector potential is obtained in the nodes of the numerical mesh. In the case of a moving coil and a deformable free surface, the magnetic field needs to be continuously recomputed with the geometrical change.

The numerical solution procedure starts with the solid metal charge at room temperature, then continuous to the melting stage and the dynamic evolution of the flow and energy transport until a quasi-steady state is achieved. In the majority of the calculations initial preheat of the aluminum to $973 \mathrm{~K}\left(700{ }^{\circ} \mathrm{C}\right)$ is used in order to speed up the procedure.

Typical quasi-steady state flow fields are shown in Figure 2. The left picture shows the induced stirring generated by the side cylindrical heating coil. A single toroidal vortex dominates the flow field and the free surface develops a pronounced dome shape due to the radially compressive Lorentz force. Heat is generated by the induced current and lost to the surroundings by radiation on the top surface and to the walls according to wall loss functions. The EM mixing maintains a temperature differential between the top and bottom of the melt, where solidification starts if the side coil heating is optionally switched off.

The left picture in Figure 2 shows the difference in melt behavior as the top coil is gradually lowered into the crucible. The top surface is depressed by electromagnetic repulsion - so contact with the melt is avoided under normal operating conditions! Mixing is now enhanced (maximum velocity grows from 0.1 to $0.8 \mathrm{~m} /$ s) with the appearance of a strong vortex surrounding the region closest to the coil. This would also be the 
region of strongest vibration leading to a sound field equivalent to that induced by an immersed sonotrode, but without metal contact. Since the two coils have different functions they can operate at different frequencies, in this case 2 and $10 \mathrm{kHz}$. The $10 \mathrm{kHz}$ value was selected to reproduce a $20 \mathrm{kHz}$ vibration, typical of sonotrode ultrasonic treatment as explained below.

\section{A. Theoretical Analysis for the Time-Dependent EM Force in $1 d$}

To demonstrate the approach used to extract the sound source, we consider the case of a long cylindrical crucible coaxially surrounded by a cylindrical coil. Since the cylinder radius is much larger than the EM skinlayer depth $\delta$, the wall can be approximated to a flat surface. Then locally, the harmonic AC magnetic field due to the frequency $\omega$ can be represented as

$$
\boldsymbol{B}=B_{\mathrm{R}} \cos \omega t+B_{\mathrm{I}} \sin \omega t,
$$

where

$$
B_{\mathrm{R}}=B_{\mathrm{o}} e^{-\frac{x}{\delta}} \cos \frac{x}{\delta} ; \quad B_{\mathrm{I}}=B_{\mathrm{o}} e^{-\frac{x}{\delta}} \sin \frac{x}{\delta},
$$

$x$ is the coordinate measured from the external wall inwards into the conducting medium. The associated AC current in the liquid is

$$
\begin{gathered}
J=J_{\mathrm{R}} \cos \omega t+J_{\mathrm{I}} \sin \omega t \\
J_{\mathrm{R}}=\sigma \frac{\omega}{2} \delta\left(B_{\mathrm{R}}+B_{\mathrm{I}}\right) ; \quad J_{\mathrm{I}}=\sigma \frac{\omega}{2} \delta\left(-B_{\mathrm{R}}+B_{\mathrm{I}}\right) .
\end{gathered}
$$

Then, the Lorentz force on liquid is

$$
\boldsymbol{F}=\boldsymbol{J} \times \boldsymbol{B}=\bar{F}+\tilde{F},
$$

which can be decomposed in the time-averaged force over a period:

$$
\bar{F}=\frac{1}{2}\left(J_{\mathrm{R}} B_{\mathrm{R}}+J_{\mathrm{I}} B_{\mathrm{I}}\right)=\frac{1}{2 \mu \delta} B_{\mathrm{o}}^{2} e^{-2 \frac{x}{\delta}}
$$

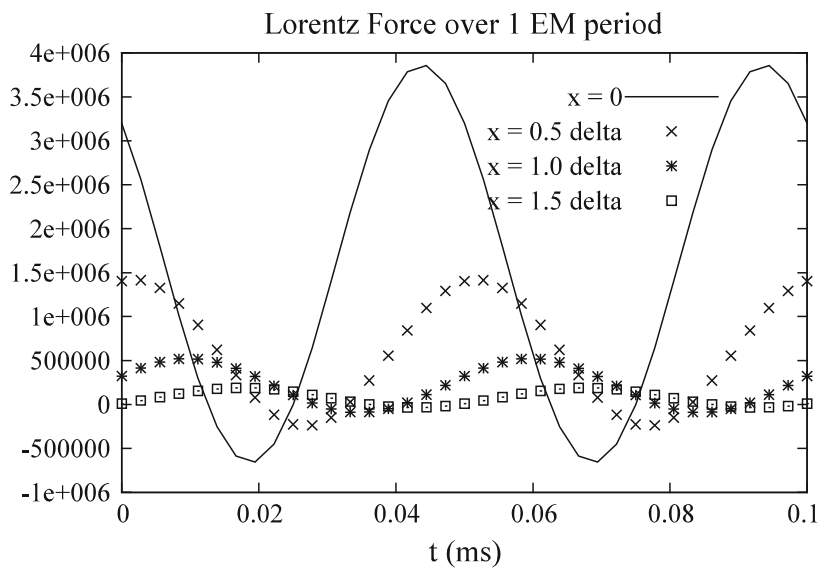

Fig. 3-EM force in liquid $\mathrm{Al}$ at four positions away from the surface at $20 \mathrm{kHz}$. and the time-dependent component:

$$
\tilde{F}=\frac{1}{2 \mu \delta} B_{\mathrm{o}}^{2} e^{-2 \frac{x}{\delta}} \sqrt{2} \cos \left(2 \omega t-2 \frac{x}{\delta}+\frac{\pi}{4}\right) .
$$

Note, the oscillating part of the Lorentz force doubles the supply frequency. The resulting sinusoidal force is shown graphically in Figure 3. It is evident that the force is only significant in the outermost part of the skin layer, decaying rapidly away from the wall at a frequency of $20 \mathrm{kHz}$. There is also a gradual change of phase through the skin layer.

The observations from this idealized 1D problem are valid in a realistic crucible simulation as shown in Figure 4, which shows the instantaneous force under the top coil and the pressure variations induced in the top of the crucible. The signal decays rapidly away from the surface and walls. The time-averaged part of the force field was used to model the incompressible flow and temperature distribution shown in Figure 2. The timedependent part of the force field is then coupled with a simplified compressible fluid model for the sound wave simulation as shown in the following section.

\section{EM SOUND GENERATION}

The sinusoidal excitation provided by the Lorentz force is the source of vibration, hence sound in the liquid.

\section{A. Linearized Euler Equations for Sound in Liquids}

The generation of sound waves by the alternating magnetic force was simulated using a Computational Acoustics approach. ${ }^{[15]}$ The equation of mass continuity and the simplified momentum equations (compressible, but no convection or viscous terms), yield the following equations:

$$
\frac{\partial p}{\partial t}+\rho c^{2} \frac{\partial \tilde{v}}{\partial x}=0 ; \quad \rho \frac{\partial \tilde{v}}{\partial t}+\frac{\partial p}{\partial x}=f ; \quad f=|\mathbf{F}|-\bar{F}
$$

This system of 1 st order partial differential equations was discretized onto a fully-staggered mesh formed of the spatial axis $x$ and the temporal axis $t$. The acoustic pressure values $p$ were stored on the cell faces of this mesh and the acoustic velocity perturbations $\tilde{v}$ were in the cell centers in the middle of each timestep - this leads to the 'leap frog' numerical scheme shown in Figure 5. ${ }^{[15]}$ The time-dependent component of the force $f$ per unit volume from [14] was evaluated in the ' $v$ ' positions and added to the right-hand side of the momentum equation. The scheme is 4 th order accurate.

\section{SOUND FIELD RESULTS}

In the following figures, samples of computed 3dimensional sound fields obtained in the aluminum crucible of Figure 2 using side and top coils are shown. Sound fields are compared with those obtained from a traditional immersed sonotrode operating at $20 \mathrm{kHz}$. Fast 

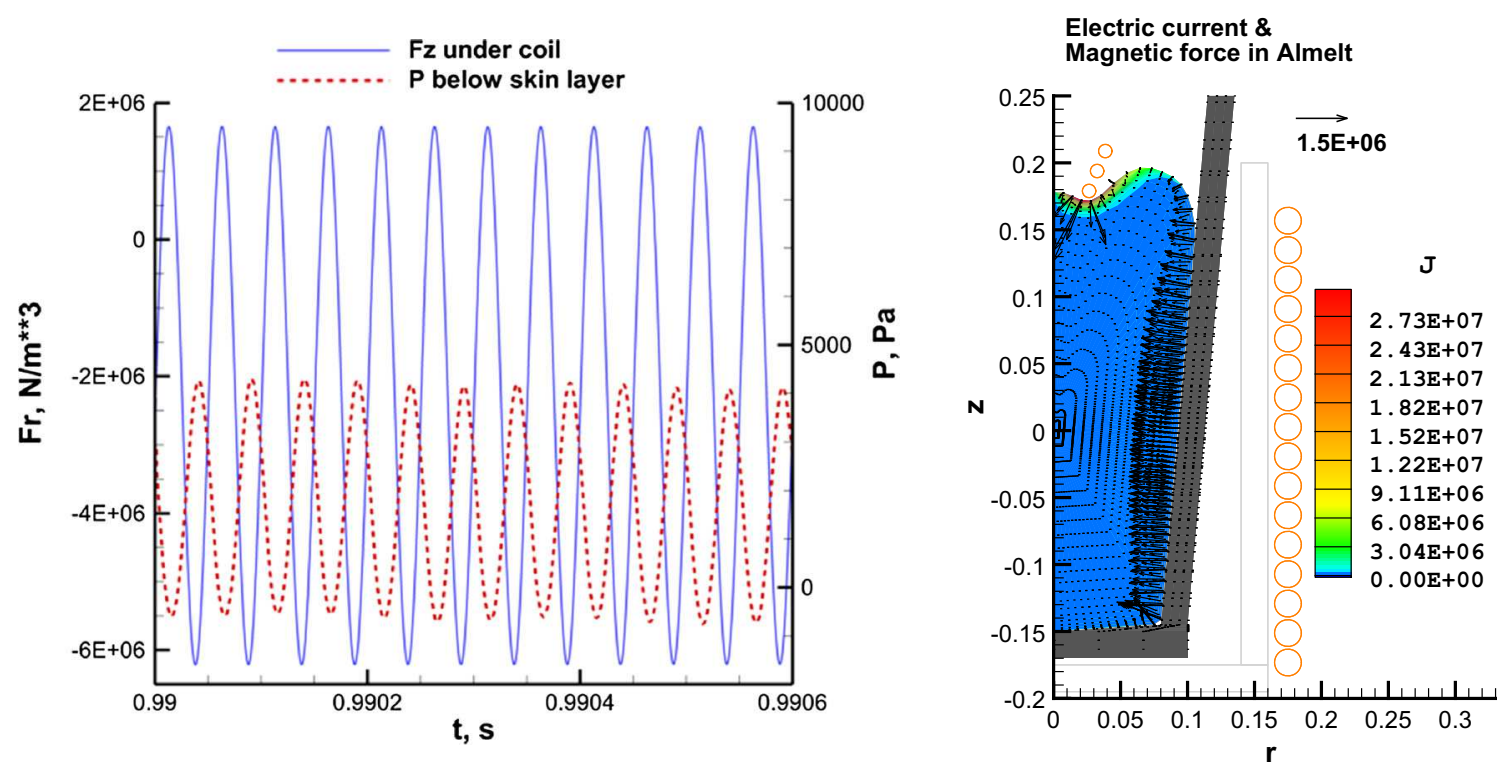

Fig. 4-Instantaneous force and pressure in the skin layer of the top coil: induced field (left) and the induced electric current and force distribution from both coils in the melt (right).
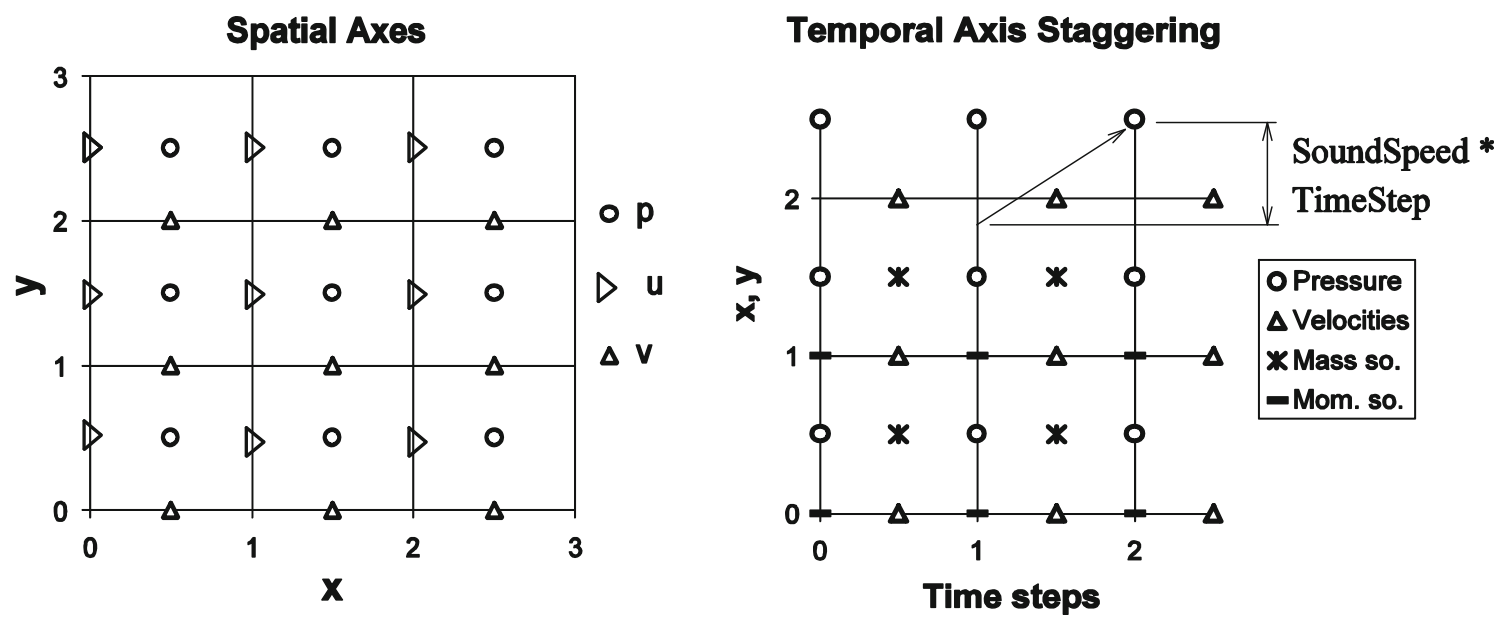

Fig. 5-Staggered grid discretization for sound calculations.

Fourier Transform (FFT) analysis of the pressure signals at various locations in the crucible allows comparison of numerical simulations against analytic solutions.

Figure 6 shows a typical pressure map excited by the top coil at an AC frequency of $10 \mathrm{kHz}$. The acoustic boundary conditions at the walls and the bottom of the crucible are perfectly reflecting, while the boundary condition at the top free surface is a 'zero pressure', which causes inversion of the pressure of the reflected wave.

The instantaneous pressure map reveals alternating high and low pressure regions at a particular timestep. Several frequencies are excited by the input signal as can be seen more clearly by the pressure trace along the axis of the crucible. Figure 7 shows the frequency content of the signal, obtained by FFT analysis. The strongest peak is at the driving frequency, $20 \mathrm{kHz}$. However, there are several natural harmonics excited in the range between 3 and $50 \mathrm{kHz}$. The corresponding peaks are given in Table I. There is a striking correspondence between the numerical and theoretical values, obtained for the updown mode of oscillation according to the relationship $f=\frac{n c}{4 L} ; \quad n=1,3,5, \ldots$, where $c$ is the speed of sound (assumed $4500 \mathrm{~m} / \mathrm{s}$ ) and $L$ is the mean height of the liquid metal level (assumed $L=0.33 \mathrm{~m}$ ). Considering the vessel is slightly conical and the resolution of this particular FFT implementation is $97 \mathrm{~Hz}$, the agreement is quite satisfactory.

Resonance can be easily reached by frequency tuning to achieve pressure thresholds relevant for cavitation. Recognizing that uncontrolled increase in amplitude may damage the crucible, a near-resonant condition may be preferable, indicated by the characteristic beat (at \pm 10 pet frequency shift) shown in Figure 8 .

It is instructive to compare the contactless and the immersed sonotrode sound fields in the same geometry, 


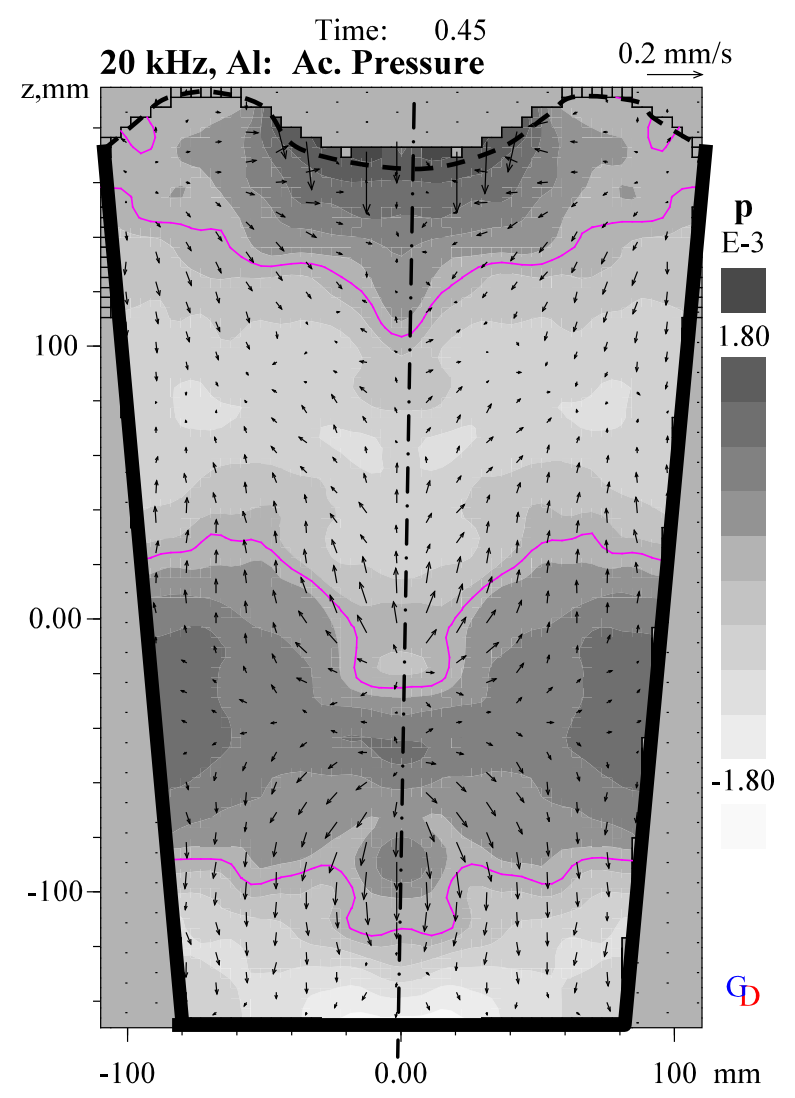

(a)

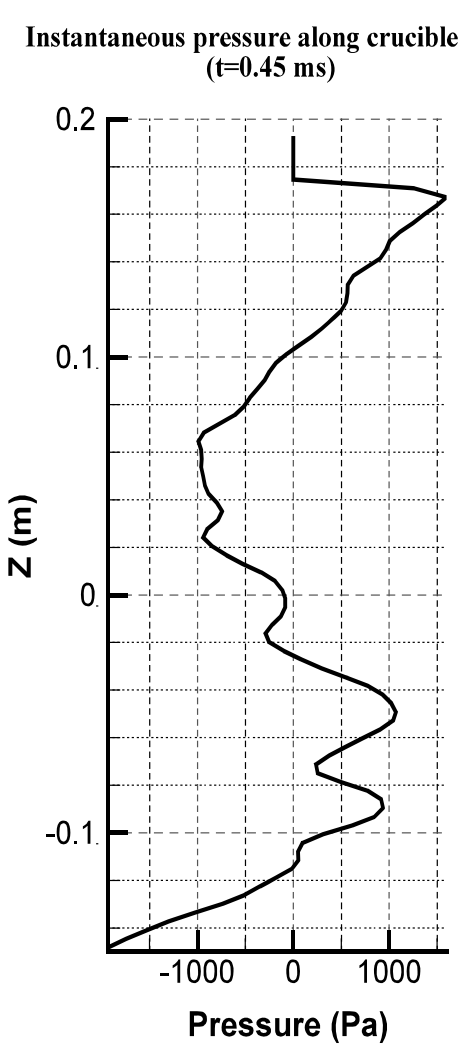

(b)

Fig. 6-Top coil active (a) Pressure contours showing alternating regions of low and high pressure in the crucible, caused by the action of mainly horizontally oriented waves $(b)$ Corresponding pressure variation along the crucible centerline.

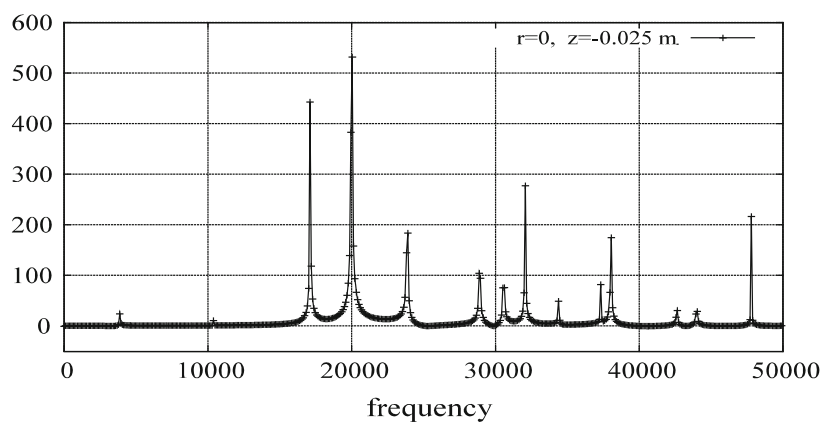

Fig. 7-Frequency spectrum of the sound signal at a central location in the crucible.

and that is demonstrated in Figure 9. The general patterns of peaks and troughs are similar, produced by standing waves generated by reflection from the crucible walls in a horizontal mode and the base and free surface in a vertical mode. It can also be seen that at the settings given the vibrating sonotrode produces higher pressure amplitudes, by a factor of three.

Changing the AC supply frequency can alter the sound field quite dramatically, since the wavelength can couple preferentially to either the longitudinal, or the lateral dimensions of the vessel. Figure 10, shows the substantial difference in sound pressure patterns between $1 \mathrm{kHz}$ - good for mixing, and $20 \mathrm{kHz}$, thought to be best for cavitation. Furthermore, the $20 \mathrm{kHz}$ case produces acoustic pressure levels that are higher by two orders of magnitude, firmly in the cavitation level regime.

Figure 11 depicts the sound field generated by the immersed coil (RHS) as well as the surface depression and stirring caused by its presence (LHS). The middle pictures show the sound 'beat' caused by the near-resonant condition at two different locations in the core of the crucible with the figure below showing how the driving frequency excites resonance at two adjacent peaks. These peaks are due to the slightly conical form of the crucible and disappear when the crucible becomes cylindrical.

\section{CONCLUSIONS}

It has been shown through a combination of MHD and Computational Acoustic simulations that EM induction can be used as a contactless replacement of the mechanical sonotrode. Then,

1. the mean EM force acts in a thin 'skin' layer and promotes bulk mixing (which a mechanical sonotrode does not!);

2. the sinusoidal part of the force is responsible for the sound waves produced;

3. the AC frequency can be adjusted to produce nearresonance pressure amplitudes for cavitation (e.g., so as to disperse particle clusters); and 
Table I. Comparison of Theoretical and Numerical Harmonics Excited by the Top Coil Operating at $20 \mathrm{kHz}$ in Aluminum

\begin{tabular}{lccccrr}
\hline$n$ & 1 & 3 & 5 & 7 & 9 & 11 \\
\hline$f$ Theoretical & 3409 & 10,227 & 17,045 & 23,864 & 30,682 & 37,500 \\
$f$ Numerical & 3860 & 10,387 & 17,097 & 23,899 & 30,609 & 37,319 \\
\hline
\end{tabular}
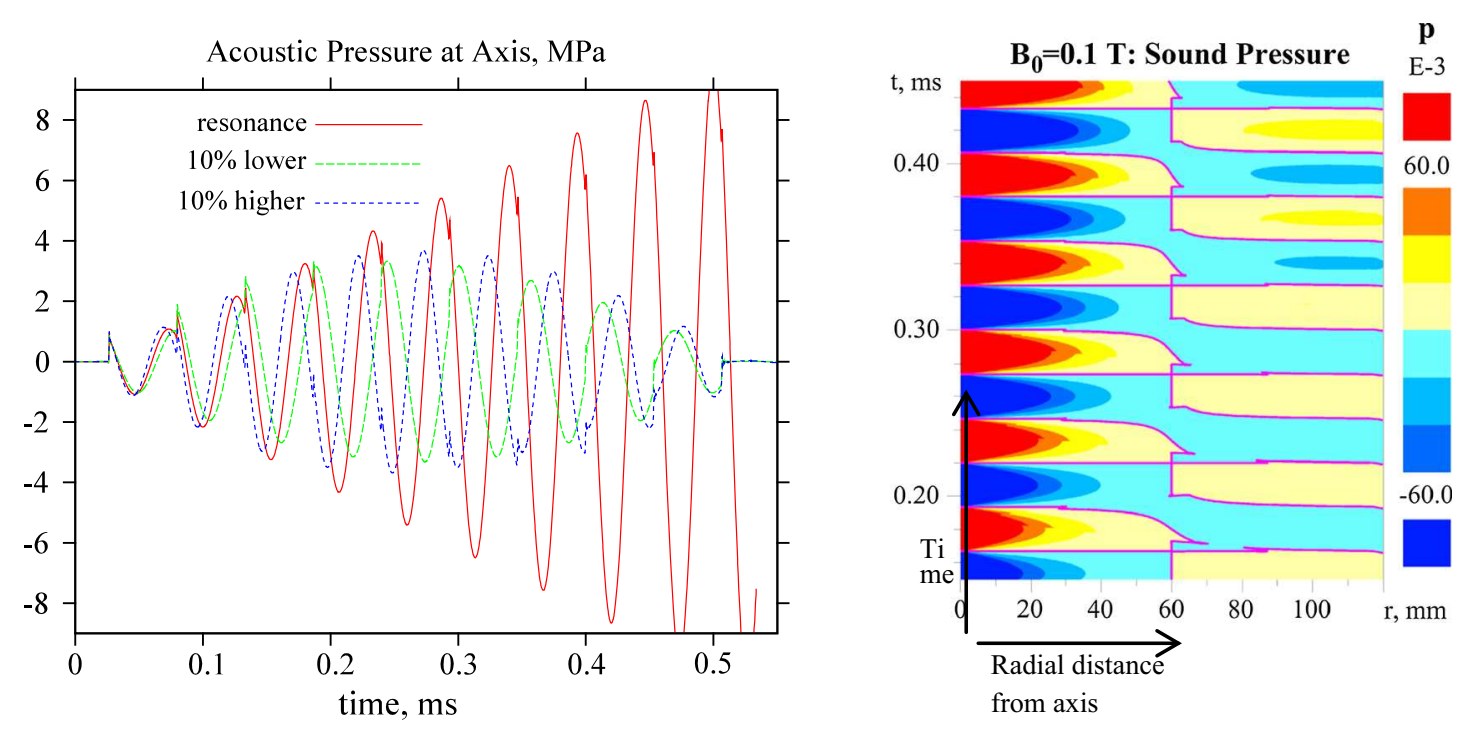

Fig. 8-Near-resonance operation with 10 pct frequency shift (left) of cylindrical aluminum crucible, showing the development of standing waves (right).

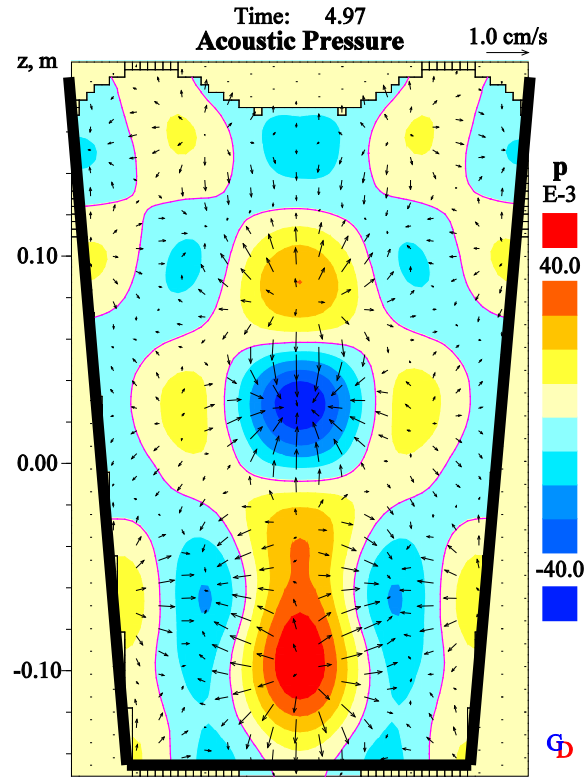

Top coil

Scale: $\pm 50 \mathrm{kPa}$

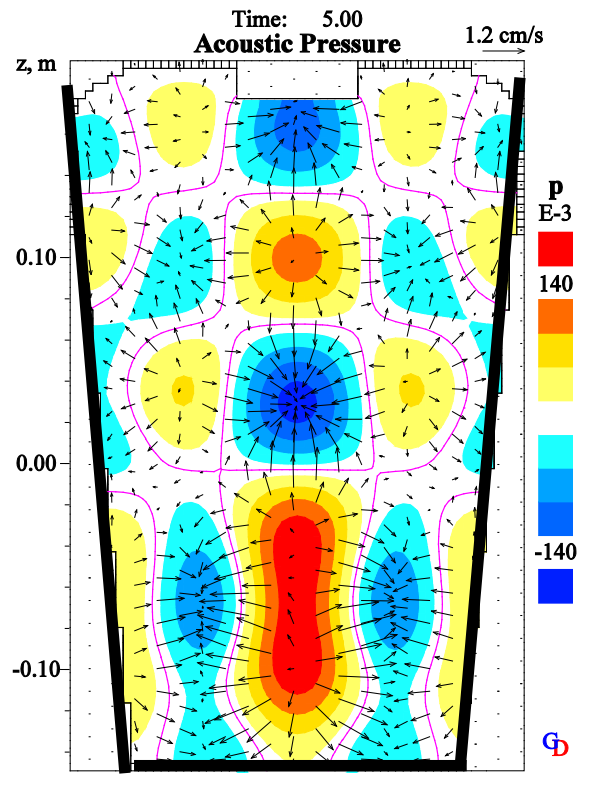

Sonotrode

Scale: $\pm 150 \mathrm{kPa}$

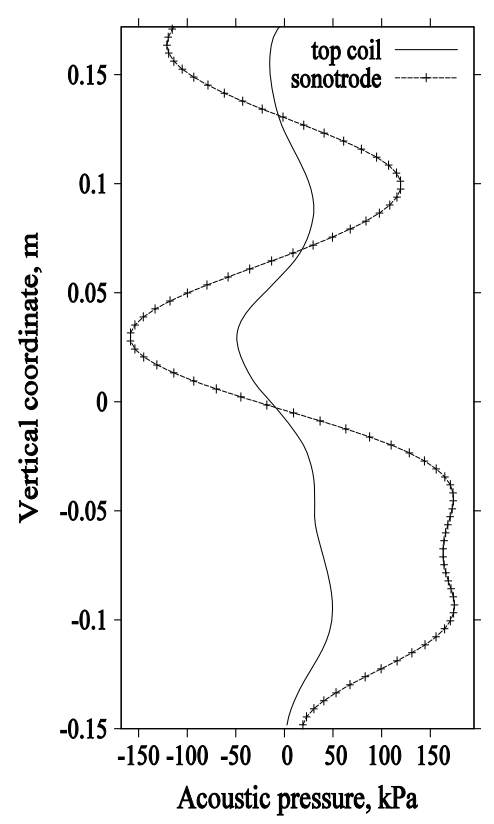

Fig. 9-Comparison of $20 \mathrm{kHz}$ sound fields generated by the top coil (left) with those of traditional immersed sonotrode (middle). The graph on the right compares the variation of the sound pressure along the central axis of the crucible.

4. the geometry of the vessel (and liquid level) will determine the pattern of sound waves produced and the resonant modes excited.
The EM "sonotrode" concept is contactless, therefore,

1. can be used for high temperature metals $(\mathrm{Ni}, \mathrm{Ti}$, etc.) with a water-cooled coil, 

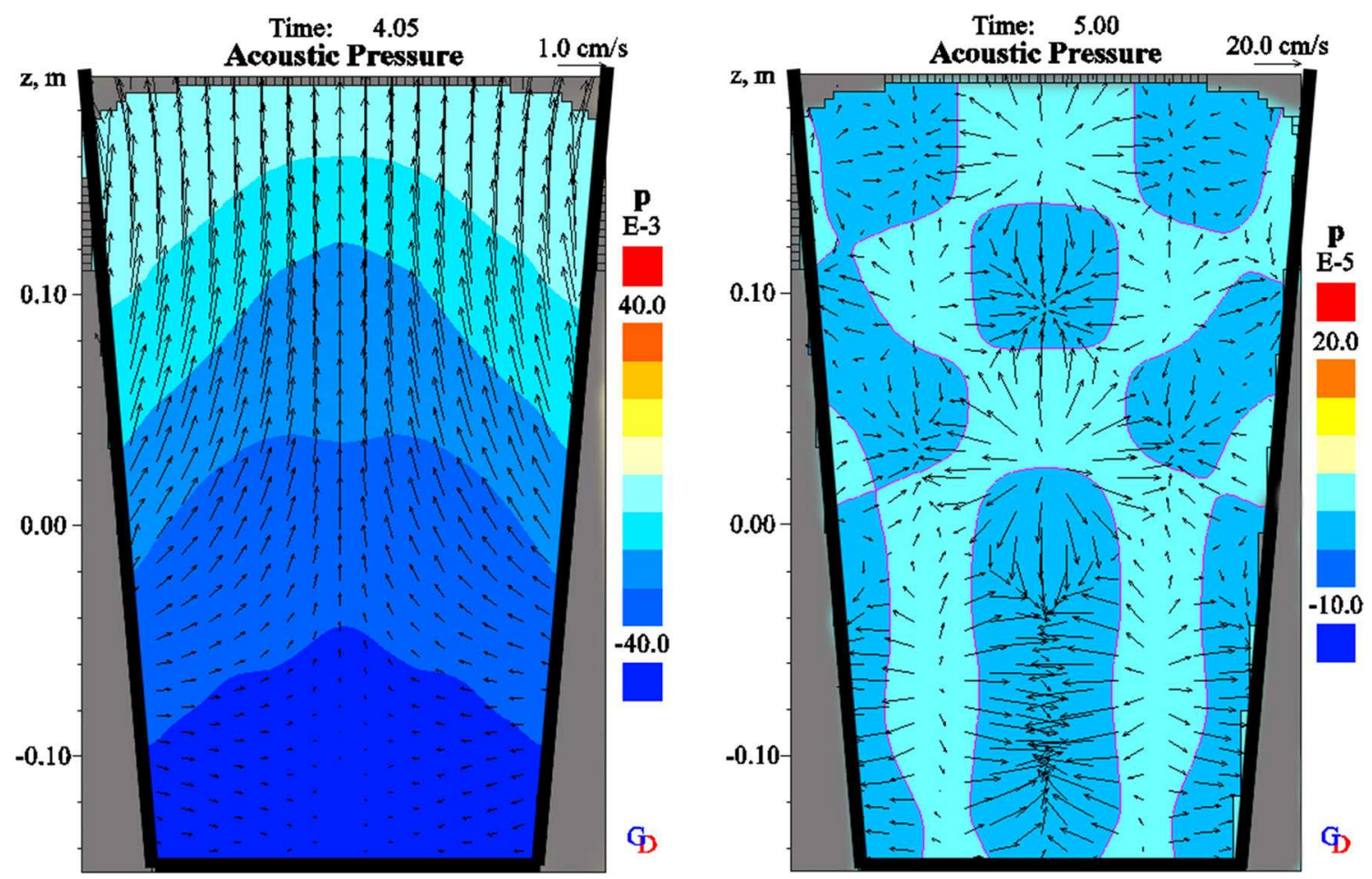

Fig. 10-EM Acoustic field, the difference between $1 \mathrm{kHz}$ (left) and $20 \mathrm{kHz}$ (right).

(a)

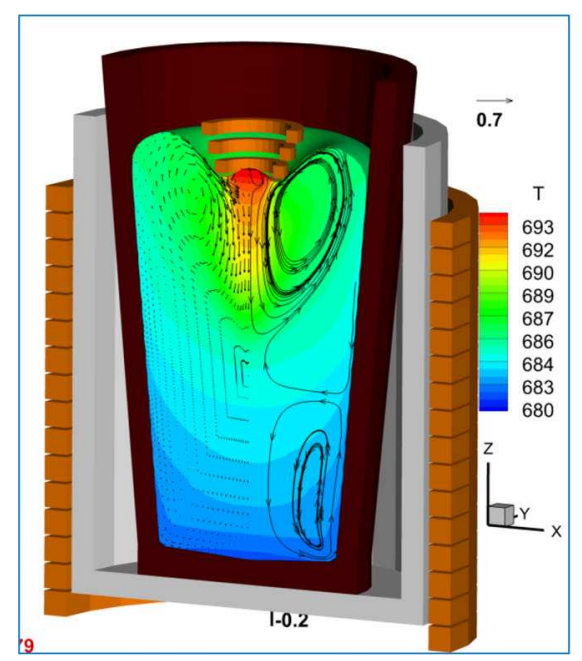

(b)

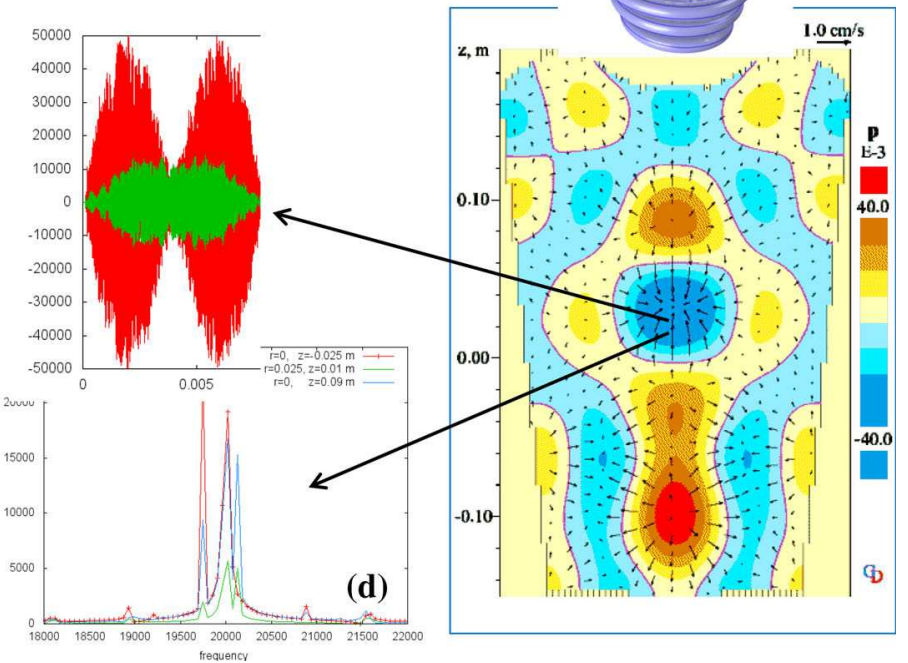

Fig. 11-(a) Crucible, inductive melt heating and stirring, (b) Sound wave 'beat' as forcing frequency approaches resonance, $(c)$ Acoustic pressure spectrum - multiple peaks due to conical crucible design, (d) Instantaneous sound field. 
2. avoids contamination of melt,

3. can fit in with existing induction coil arrangements, and

4. a practical implementation in the form of an immersed top coil has been shown to mimic the operation of an immersed sonotrode.

Work is currently in progress to test this setup experimentally, within the framework of the EU-funded project ExoMet, for the production of light alloy metal matrix nano-composites (MMNCs).

\section{ACKNOWLEDGMENTS}

The authors acknowledge financial support from the ExoMet Project (co-funded by the European Commission (contract FP7-NMP3-LA-2012-280421), by the European Space Agency and by the individual partner organizations).

\section{REFERENCES}

1. J. Campbell: Int. Met. Rev., 1981, vol. 26 (2), pp. 71-108.

2. O. Abramov: Ultrasound in Liquid and Solid Metals, CRC Press, Boca Raton, FL, 1994, p. 289.
3. G.I. Eskin and D.G. Eskin: Ultrason. Sonochem., 2003, vol. 10 (4-5), pp. 297-301.

4. W.W. Xu, I. Tzanakis, P. Srirangam, S. Terzi, W.U. Mirihanage, D.G. Eskin, and P.D. Lee: In situ Synchrotron Radiography of Ultrasound Cavitation in a Molten Al-10Cu Allo y; TMS2015 144th Annual Meeting \& Exhibition; 15-19 March, 2015, in press.

5. C. Vives: J. Cryst. Growth, 1996, vol. 158 (1-2), pp. 118-27.

6. A. Verma, S.P. Tewari, and J. Prakash: Int. J. Eng. Sci. Technol., 2011, vol. 3 (6), pp. 5215-20.

7. J. Dong, J. Cui, X. Zeng, and W. Ding: Mater. Trans., 2005, vol. 46 (1), pp. 94-99.

8. G. Djambazov, V. Bojarevics, B. Lebon, and K. Pericleous: Contactless Acoustic Wave Generation in a Melt by Electromagnetic Induction, TMS Annual Meeting, Light Metals 2014, John Grandfield, eds., TMS, 2014, pp. 1379-82.

9. K Pericleous and V Bojarevics: Progr. Comput. Fluid Dyn., 2007, vol. $7(2 / 3 / 4)$, pp. $118-27$.

10. V. Bojarevics, R.A. Harding, K.A. Pericleous, and M. Wickins: Metall. Mater. Trans. B, 2004, vol. 35B, pp. 785-803.

11. H Alehossein and Z Qin: Int. J. Numer. Methods Eng., 2007, vol. 72 , pp. $780-807$.

12. G.S.B. Lebon, K. Pericleous, I. Tzanakis, and D. Eskin: Advances in the Science and Engineering of Casting Solidification: An MPMD Symposium Honoring Doru Stefanescu, in press.

13. D.C. Wilcox: Turbulence Modelling for $C F D$, 2nd ed., DCW Industries, California, 1998

14. A. Bojarevics, V. Bojarevics, J. Gelfgat, and K. Pericleous: Magnetohydrodynamics, 1999, vol. 35 (3), pp. 258-77.

15. G.S. Djambazov, C.H. Lai, and K.A. Pericleous: AIAA J., 2000, vol. 38 (1), pp. 16-21. 